\title{
Remarks about the Tensor Mode Detection by the BICEP2 Collaboration and the Super-Planckian Excursions of the Inflaton Field
}

\author{
A. Kehagias ${ }^{1}$ and A. Riotto ${ }^{1}$ \\ ${ }^{1}$ Département de Physique Théorique and Centre for Astroparticle Physics (CAP), \\ Université de Genève, 24 quai E. Ansermet, CH-1211 Geneva, Switzerland
}

(Dated: June 26, 2018)

\begin{abstract}
The recent detection by the BICEP2 collaboration of a high level of tensor modes has relevant implications which we briefly discuss in this short note. In particular, the large angle CMB Bmode polarisation seems to imply problematic super-Planckian excursions of the inflaton field. We provide some comments about this point and in particular we stress a natural resolution to it: given our current (and probably future) observational ignorance about the true source of the scalar perturbations, one should abandon the theoretical prejudice that they are associated to the inflaton fluctuations.
\end{abstract}

Inflation [1, 2] has become the dominant paradigm for understanding the initial conditions for structure formation and for Cosmic Microwave Background (CMB) anisotropy. In the inflationary picture, primordial density and gravity-wave fluctuations are created from quantum fluctuations "redshifted" out of the horizon during an early period of superluminal expansion of the universe, where they are "frozen" 3,4$]$.

The recent measurement of the tensor modes from large angle CMB B-mode polarisation by BICEP2 [5], implying a tensor-to-scalar ratio

$$
r=0.2_{-0.05}^{+0.07},
$$

has put inflation on a ground which is firmer than ever. Indeed, the generation of gravity-wave fluctuations is a generic prediction of an accelerated de Sitter expansion of the universe. The tensor modes may be viewed as ripples of spacetime around the background metric

$$
g_{\mu \nu}=\mathrm{d} t^{2}-a^{2}(t)\left(\delta_{i j}+h_{i j}\right) \mathrm{d} x^{i} \mathrm{~d} x^{j},
$$

where $a$ is the scale factor and $t$ is the cosmic time. The tensor $h_{i j}$ is traceless and transverse and has two polarizations, $\lambda= \pm$. Since gravity-wave fluctuations are (nearly) frozen on super-Hubble scales, a way of characterizing them is to compute their spectrum on scales larger than the Hubble radius during inflation. The power spectrum of gravity-wave modes turns out to be

$$
\mathcal{P}_{T}(k)=\frac{8}{M_{p}^{2}}\left(\frac{H_{*}}{2 \pi}\right)^{2}\left(\frac{k}{a H_{*}}\right)^{-2 \epsilon},
$$

where $M_{p}=(8 \pi G)^{-1 / 2} \simeq 2.4 \times 10^{18} \mathrm{GeV}$ is the Planck scale. Here $\epsilon=\left(\dot{\phi}^{2} / 2 M_{p}^{2} H_{*}^{2}\right)$ is a standard slow-roll parameter and $H_{*}=\dot{a} / a$ indicates the Hubble rate during inflation.
On the other hand, the power spectrum of curvature perturbations in slow-roll inflationary models is given by

$$
\mathcal{P}_{\zeta}(k)=\frac{1}{2 M_{p}^{2} \epsilon}\left(\frac{H_{*}}{2 \pi}\right)^{2}\left(\frac{k}{a H_{*}}\right)^{n_{\zeta}-1},
$$

where $n_{\zeta} \simeq 1$ is the spectral index. Since the fractional changes of the power spectra with scales are much smaller than unity, one can safely consider the power spectra as roughly constant on the scales relevant for the $\mathrm{CMB}$ anisotropy and define a tensor-to-scalar amplitude ratio

$$
r=\frac{\mathcal{P}_{T}}{16 \mathcal{P}_{\zeta}}=\epsilon .
$$

The recent BICEP2 dataset allows to extract the value of the Hubble rate during inflation to be

$$
H_{*} \simeq 1.1 \times 10^{14} \mathrm{GeV},
$$

corresponding to an energy scale during inflation $V^{1 / 4}$ of about $2 \times 10^{16} \mathrm{GeV}$, astonishingly closed to the scale of grand-unification in the minimal supersymmetric extension of the standard model of weak interactions.

Let us now pause for a moment and summarize what we really know about the properties of the scalar and tensor perturbations generated during inflation:

- First of all, the recent Planck data [6] tell us that the scalar perturbations have an almost scaleinvariant spectrum and are of the adiabatic type, but we do not know the real source of the scalar perturbations. This point will be relevant below.

- The scalar perturbations are nearly-Gaussian or, in any case, the level of non-Gaussianity, parametrized by the non-linear parameter $f_{\mathrm{NL}}$, is severely constrained [7]. 
- The energy scale of inflation is approximately the grand-unification scale.

These are consequences of the observational facts which nobody can dispute. Here are some implications one can draw:

- In the sudden reheating approximation, the maximum reheating temperature after inflation of about

$$
\begin{aligned}
T_{\mathrm{RH}} & =\left(\frac{30 V}{\pi^{2} g_{*}\left(T_{\mathrm{RH}}\right)}\right)^{1 / 4} \\
& \simeq 5.6\left(\frac{10^{3}}{g_{*}\left(T_{\mathrm{RH}}\right)}\right)^{1 / 4} \times 10^{15} \mathrm{GeV} .
\end{aligned}
$$

The true reheating temperature is likely to be smaller; one should also remember that at temperatures larger than about $2.4 \times 10^{14} \mathrm{GeV}$ the universe is not in thermal equilibrium and one may not define a temperature [8].

- The Standard Model Higgs field $h$ needs to be nontrivially coupled either to the inflaton field or to gravity. Indeed, for a Higgs mass in the range $(124-126) \mathrm{GeV}$, and for the current central values of the top mass and strong coupling constant, the Higgs potential develops an instability around $10^{11}$ $\mathrm{GeV}$, see for instance Ref. [9]. As this instability scale is much smaller than $H_{*}$, the classical value of the Higgs field will be easily pushed above the instability point by its fluctuations during inflation [10]. This can be avoided by either coupling the Higgs field to the Ricci scalar, $\xi R h^{\dagger} h$ with $\xi \gtrsim 10^{-1}$, or to the inflaton itself in order to suppress the Higgs fluctuations during inflation.

- Similar remarks can be drawn for the case in which supersymmetry is a (broken) symmetry of nature. Indeed, there are many flat directions in the field space of low-energy supersymmetric models. It may happen that some combination of the squark and/or slepton mass squared parameters get negative at some scale below the Planck scale when running through the renormalization group equations from the weak scale up. This may happen if the sfermion masses are lighter than the gaugino masses, leading either to the appearance of unacceptable color/charge breaking unbounded from below directions in the effective potential for the squark and/or slepton fields. The instability case can be again smaller than $H_{*}$, posing a threat during inflation due to the large fluctuations of the sfermion fields [11]. Again, one is led to conclude that low-energy supersymmetric partners must be coupled to the inflation field.
Furthermore, and maybe more interestingly, the recent detection of a high level of tensor modes have generated a lot of surprise based on the following argument due to Lyth [12].

If the scalar perturbations are ascribable to only one scalar degree of freedom, the inflaton field itself (this is not a gauge-invariant statement), then the slow-roll paradigm gives, using the definition of $\epsilon$ and Eq. (5),

$$
\frac{1}{M_{p}}\left|\frac{\mathrm{d} \phi}{\mathrm{d} N}\right|=\sqrt{2} r^{1 / 2}
$$

where $\mathrm{d} \phi$ is the change in the inflaton field in $\mathrm{d} N=$ $H \mathrm{~d} t \simeq \mathrm{d} \ln a$ Hubble times. While the scales corresponding to the relevant multipoles in the CMB anisotropy are living the Hubble radius $\Delta N \simeq 4.6$ and therefore the field variation is

$$
\frac{\Delta \phi}{M_{p}} \simeq\left(\frac{r}{2 \times 10^{-2}}\right)^{1 / 2}
$$

This is a minimum estimate because inflation continues for some number $N$ of $e$-folds of order of 50 . The detection of gravitational waves requires in general variation of the inflaton field of the order of the Planck scale [12].

This conclusion is considered to be a problem as slowroll models of inflation are generically based on fourdimensional field theories, possibly involving supergravity, where higher-order operators with powers of $\left(\phi / M_{p}\right)$ are disregarded. This assumption is justified only if the inflaton variation is small compared to the Planck scale. It is therefore difficult to construct a satisfactory model of inflation firmly rooted in modern particle theories having possibly supersymmetry as a crucial ingredient and with large variation of the inflaton field.

It is more than fair to say that, based on this argument, there was a strong theoretical prejudice against the likelihood of observation of gravity-waves. So, now that a high level of tensor modes have been observed, where do we stand? Do we still believe that Planckian excursions of the inflaton field is a threat?

There are at least three arguments we may offer in favour of a more relaxed attitude.

The first one is in fact quite simple: it is a theoretical prejudice that the scalar perturbations come from the inflaton field.

The sad reality is that we have no idea what is the real source of the scalar perturbations during inflation. Even worse, in the absence of a detection of a large nonGaussianity, we will probably never know.

The problem of having large excursion of the inflaton field arises only if the scalar perturbations are generated by the inflaton itself, which is the origin of the relation (8). Despite the simplicity of the inflationary paradigm, 
the mechanism by which cosmological adiabatic perturbations are generated is not yet established. It is conceivable that the total curvature perturbation $\zeta$ is not a constant (in time) on super-Hubble scales, but on the contrary changes on arbitrarily large scales due to a nonadiabatic pressure perturbation which may be present extra (other than the inflaton) degrees of freedom are present. While the entropy perturbations evolve independently of the curvature perturbation on large scales, the evolution of the large-scale curvature is sourced by the entropy perturbation $\delta S$

$$
\dot{\zeta} \sim \delta S
$$

A realization of this mechanism is represented, for instance, by the curvaton mechanism [13 16] where the final curvature perturbations are produced from an initial isocurvature perturbation associated to the quantum fluctuations of a light scalar field (other than the inflaton), the curvaton, whose energy density is negligible during inflation. The curvaton isocurvature perturbations are transformed into adiabatic ones when the curvaton decays into radiation much after the end of inflation. Other mechanisms for the generation of cosmological perturbations have been proposed, for instance the modulated decay scenario [17 19], where super-Hubble spatial fluctuations in the decay rate of the inflaton field are induced during inflation, causing adiabatic perturbations in the final reheating temperature in different regions of the universe. Also, the dominant contribution to the primordial curvature perturbation may be generated at the end of inflation [20, 21].

Consider, for instance, the simplest curvaton scenario [14], being $\sigma$ the curvaton field. During inflation, the curvaton energy density is negligible and isocurvature perturbations with a flat spectrum are produced in the curvaton field $\sigma,\left\langle\delta \sigma^{2}\right\rangle^{\frac{1}{2}}=\left(H_{*} / 2 \pi\right)$, where $\sigma_{*}$ is the value of the curvaton field during inflation. After the end of inflation, the curvaton field oscillates during some radiationdominated era, causing its energy density to grow and thereby converting the initial isocurvature into curvature perturbation. After the curvaton decays $\zeta$ becomes constant. In the approximation that the curvaton decays instantly it is then given by $\zeta \simeq(2 \gamma / 3)(\delta \sigma / \sigma)_{*}$, where $\gamma \equiv\left(\rho_{\sigma} / \rho\right)_{D}$ and the subscript $D$ denotes the epoch of decay. The corresponding spectrum is [14]

$$
\mathcal{P}_{\zeta}^{\frac{1}{2}} \simeq \frac{2 \gamma}{3}\left(\frac{H_{*}}{2 \pi \sigma_{*}}\right)
$$

Since the amplitude of curvature perturbation $\mathcal{P}_{\zeta}^{1 / 2}$ must match the observed value $5 \times 10^{-5}$, from Eq. (11) one infers that

$$
\sigma_{*} \simeq 2 \gamma \times 10^{3} H_{*}
$$

For $10^{-1} \lesssim \gamma \lesssim 1$, the corresponding level of nonGaussianity is such that $-5 / 4 \lesssim f_{\mathrm{NL}} \lesssim 5 / 4 r$ [22]. Since a level of (local) non-Gaussianity compatible with the present Planck data is $f_{\mathrm{NL}} \lesssim 10$ [22], we conclude that

$$
\left(2 \times 10^{16} \mathrm{GeV} \lesssim \sigma_{*} \lesssim 2 \times 10^{17} \mathrm{GeV}\right)
$$

This is is comfortably below the Planckian scale. Of course, we are working under the assumption that the the curvature perturbations of the inflaton field are suppressed. This may happen, for instance, if the inflaton field is well anchored at the false vacuum driving inflation with a mass much $m_{\phi}$ larger than the Hubble rate during inflation. Suppose indeed that the inflaton potential is of the form

$$
V(\phi)=V_{0}+\frac{m_{\phi}^{2}}{2} \phi^{2}+\cdots
$$

where $\phi=0$ is the location of the minimum around which $m_{\phi}^{2} \gg H_{*}^{2} \simeq V_{0} / M_{p}$. Under these circumstances, slowroll conditions are badly violated since $\eta=\left(m_{\phi}^{2} / 3 H_{*}^{2}\right) \gtrsim$ 1 and the fluctuations of the inflaton field on superHubble scales read

$$
\mathcal{P}_{\delta \phi}(k)=\left(\frac{H_{*}}{2 \pi}\right)^{2}\left(\frac{k}{a H_{*}}\right)^{3} e^{-2 m_{\phi}^{2} / H_{*}^{2}} .
$$

The resulting power spectrum is suppressed [23]. This scenario just needs an extra degree of freedom which act as a clock to remove the inflaton from its false vacuum, thus ending inflation. A red spectrum for the curvature perturbations can be easily obtained by supposing that the curvaton field during inflation is slowly rolling to its true vacuum from the top to its potential, such that its effective mass squared $\mathrm{m}^{2}$ is negative and $n_{\zeta}=1+\left(2 m^{2} / 3 H_{*}^{2}\right) \simeq 0.96$. Similar considerations are obtained in the modulated decay scenario where the inflaton decay rate $\Gamma$ depends on a light field $\sigma$ quadratically, $\Gamma \sim \sigma^{2}$. The corresponding power spectrum reads [22]

$$
\mathcal{P}_{\zeta}^{\frac{1}{2}} \simeq \frac{1}{6} \frac{\mathrm{d} \ln \Gamma}{\mathrm{d} \sigma}\left\langle\delta \sigma^{2}\right\rangle^{\frac{1}{2}}=\frac{1}{3}\left(\frac{H_{*}}{2 \pi \sigma_{*}}\right)
$$

and non-Gaussianity parameter is small, $f_{\mathrm{NL}} \simeq 5 / 2$.

Suppose though that we insist in taking a minimalistic approach and restrict ourselves to the standard scenario where the scalar perturbations are due to the inflaton itself.

The second argument of why we should maybe not worry too much about trans-Planckian excursions of the inflaton field is based on the following logic.

In order to generate Planck suppressed higherdimensional operator in the effective field theory, one 
has to integrate out degrees of freedom. Apart from the gravitons (more later), these might be heavy states (possibly with a bare Planckian mass). Consider, for instance, fermions field coupled to the inflaton through a Yukawa coupling which gives them an extra mass of the form $g \phi$, being $g$ a coupling constant.

If during inflation $\phi \gg M_{p}$, then these fermions will have trans-Planckian masses (unless $g$ is tiny). As discussed in Ref. 24], trans-Planckian massive states do not describe independent quantum degrees of freedom, but rather macroscopic classical states. The latter are then described by other light fundamental degrees of freedom, such as the massless gravitons, and in fact are just classical black holes. In other words, it is possible that all states to which the inflaton is coupled to during the inflationary phase are classical black holes. If true, this fact immediately implies that operators obtained by integrating out such trans-Planckian massive states will be exponentially suppressed at least by the Boltzmann factor $e^{-S}$, where $S \simeq g^{2} \phi^{2} / / M_{p}^{2}$ is the Bekenstein-Hawking entropy. As a consequence, dangerous higher-dimensional operators of the form $\left(\mathcal{O}_{n}=\phi^{n} / M_{p}^{n-4}\right)$ obtained after integrating out such trans-Planckian massive states are Boltzmann suppressed and enter the effective Lagrangian as

$$
\mathcal{L} \supset e^{-S} \frac{\phi^{n}}{M_{p}^{n-4}},
$$

nullifying in this way all potentially higher-dimensional operators $\mathcal{O}_{n}$.

Our final argument that trans-Planckian values of the inflaton might be harmless is based on the fact that the effective potential is actually an expansion in the treelevel potential and its second derivatives. Therefore, the anticipated expansion of the effective potential as $V_{\text {eff }}=$ $V+\sum_{n} c_{n} \phi^{n} / M_{p}^{n-4}$ might be reorganised and written as an expansion in terms of $V$ and $V^{\prime \prime}$ (primes indicating here differentiation with respect to the inflation field) as these are the physical quantities corresponding to the energy density and mass squared respectively.

This point is certainly not new and it was well-stressed, for example, by Linde in Ref. [25] (see also Ref. [26]).

To be more specific, let us consider a scalar $\phi$ coupled to gravity and write the metric fluctuations above a background with metric $\bar{g}_{\mu \nu}$ as

$$
g_{\mu \nu}=\bar{g}_{\mu \nu}+\kappa h_{\mu \nu}
$$

where $\kappa^{2}=2 / M_{p}^{2}$. Then, the quadratic part of the action turns out to be

$$
\begin{aligned}
\mathcal{L} & =-\frac{1}{4} h_{\kappa \lambda} D^{\kappa \lambda, \rho \sigma} h_{\rho \sigma}-\frac{\kappa^{2}}{4}\left(2 h^{\mu \lambda} h_{\lambda}^{\nu}-h_{\kappa}^{\kappa} h^{\mu \nu}\right) \partial_{\mu} \phi \partial_{\nu} \phi \\
& -\frac{\kappa^{2}}{16}\left(2 h^{\lambda \sigma} h_{\lambda \sigma}-\left(h_{\nu}^{\nu}\right)^{2}\right)\left(\partial_{\mu} \phi \partial^{\mu} \phi-V(\phi)\right),
\end{aligned}
$$

where $D^{\mu \nu, \kappa \lambda}$ is an invertible differential operator. The one-loop effective potential will be given by integrating out the $h_{\mu \nu}$. The result in this case is (with some corrections with respect to Ref. [27])

$$
\begin{aligned}
V_{\text {one-loop }} & =\frac{1}{2} \ln \operatorname{det}\left(\square-V^{\prime \prime}\right) \\
& +\ln \operatorname{det}\left(D^{\mu \nu, \kappa \lambda}-I^{\mu \nu, \kappa \lambda} V\right),
\end{aligned}
$$

where $I^{\mu \nu, \kappa \lambda}$ is a tensor constructed out of products of $\delta_{\mu}^{\nu}$ 's. This is a formal expression for the effective potential and it needs to be regularized with a cut-off scale (of the order of $M_{p}$ ). Using constant scalar field configurations, it is clear that after integrating out gravitons, the oneloop effective potential turns out to be a function of $V$ and $V^{\prime \prime}$. Indeed, an explicit calculation [27] reveals that the effective potential is of the form

$$
V_{\mathrm{eff}}=V(\phi)+M_{p}^{4} \sum_{n m} c_{n m} \frac{V^{\prime \prime n} V^{m}}{M_{p}^{4(n+m)}},
$$

when quantum gravity effects are taken into account. Again, there are nowhere $\phi^{n} / M_{P}^{n-4}$ terms and the inflationary predictions are not spoiled as long as $V \ll M_{p}^{4}$ and $V^{\prime \prime} \ll M_{p}^{2}$.

It may also happens that due to an undelrying symmetry, trans-Planckian values of the fields are harmless. For example, it is known that string theory on compactified on a circle of radius $R$ (or on spaces with $U(1)$ isometries in general) has the T-duality symmetry $R \rightarrow \ell_{s}^{2} / R$ where $\ell_{s}$ is the string scale, which interchanges winding and momentum states. This symmetry has a fixed point at $R=\ell_{s}$. This implies that the theory defined on a circle with radius $R>\ell_{s}$ is actually identical to a circle of radius $R<\ell_{s}$ from the string point of view. One may try to implement the same idea for the inflaton itself by residing to a similar symmetry to restrict the possible values of the inflaton to sub-Planckian region. For example, one may assume that the inflaton is part a complex field $\tau$ with a potential which is invariant under $S L(2, \mathbb{Z})$ transformations generated by $\tau \rightarrow-M_{p}^{2} / \tau$ and $\tau \rightarrow \tau+M_{p}$ 28]. In such a case, if the inflaton for example was the modulus $|\tau|$, trans-Planckian values $|\tau|>M_{p}$ are equivalent to sub-Planckian values $|\tau|<M_{p}$. However, in this case one should also ensure that $S L(2, \mathbb{Z})$ is not broken by quantum gravity effects, although it is believed that in string theory such symmetries are indeed exact.

Let us also note that one can construct class of single small field models of inflation that can predict, contrary to popular wisdom, an observable gravitational wave signal in the CMB anisotropies [29]. Finally, it might well be that the observed tensor modes generated at the linear level are subdominant with respect to those created by a spectator scalar field with speed of sound lower than unity (in such a case the spectral index $n_{T}$ of the tensor modes can be easily larger than zero) 30]. 
For sure, the detection of a large level of tensor modes from inflation will spur the community towards a better understanding of some crucial theoretical issues, possibly with some interesting connections to low-energy physics too.

[1] A. Guth, Phys. Rev. D 23, 347 (1981)

[2] D. H. Lyth and A. Riotto, Phys. Rept. 3141 (1999); A. Riotto, hep-ph/0210162 W. H. Kinney, astro-ph/0301448.

[3] V. F. Mukhanov and G. V. Chibisov, JETP Lett. 33, 532 (1981).

[4] J. M. Bardeen, P. J. Steinhardt, and M. S. Turner, Phys. Rev. D 28, 679 (1983).

[5] P. A. RAde et al. [ BICEP2 Collaboration], arXiv:1403.3985 [astro-ph.CO].

[6] P. A. R. Ade et al. [Planck Collaboration], arXiv:1303.5082 [astro-ph.CO].

[7] P. A. R. Ade et al. [Planck Collaboration], arXiv:1303.5084 [astro-ph.CO].

[8] K. Enqvist and J. Sirkka, Phys. Lett. B 314, 298 (1993) hep-ph/9304273.

[9] J. Elias-Miro, J. R. Espinosa, G. F. Giudice, G. Isidori, A. Riotto and A. Strumia, Phys. Lett. B 709, 222 (2012) arXiv:1112.3022 [hep-ph]].

[10] J. R. Espinosa, G. F. Giudice and A. Riotto, JCAP 0805, 002 (2008) arXiv:0710.2484 [hep-ph]].

[11] A. Riotto, Phys. Rev. D 86, 125038 (2012) arXiv:1211.1321 [hep-ph]].

[12] D. H. Lyth, Phys. Rev. Lett. 78, 1861 (1997).

[13] K. Enqvist and M. S. Sloth, Nucl. Phys. B 626, 395 (2002) arXiv:hep-ph/0109214.

[14] D. H. Lyth and D. Wands, Phys. Lett. B 524, 5 (2002).

[15] T. Moroi and T. Takahashi, Phys. Lett. B 522,
215 (2001) [Erratum-ibid. B $\quad \mathbf{5 3 9}, \quad 303$ (2002) arXiv:hep-ph/0110096.

[16] D. H. Lyth, C. Ungarelli and D. Wands, Phys. Rev.D 67, 023503 (2003).

[17] G. Dvali, A. Gruzinov and M. Zaldarriaga, arXiv:astro-ph/0303591

[18] L. Kofman, arXiv:astro-ph/0303614

[19] G. Dvali, A. Gruzinov and M. Zaldarriaga, Phys. Rev. D 69, 023505 (2004) astro-ph/0303591.

[20] D. H. Lyth, JCAP 0511, 006 (2005) astro-ph/0510443.

[21] D. H. Lyth and A. Riotto, Phys. Rev. Lett. 97, 121301 (2006) astro-ph/0607326.

[22] N. Bartolo, E. Komatsu, S. Matarrese and A. Riotto, Phys. Rept. 402, 103 (2004) astro-ph/0406398.

[23] G. Dvali and S. Kachru, In *Shifman, M. (ed.) et al.: From fields to strings, vol. $2^{*}$ 1131-1155 hep-th/0309095; L. Pilo, A. Riotto and A. Zaffaroni, JHEP 0407, 052 (2004) hep-th/0401004; L. Pilo, A. Riotto and A. Zaffaroni, Phys. Rev. Lett. 92, 201303 (2004) astro-ph/0401302.

[24] G. Dvali and C. Gomez, arXiv:1005.3497 [hep-th].

[25] A. D. Linde, Contemp. Concepts Phys. 5, 1 (1990) hep-th/0503203.

[26] N. Kaloper and L. Sorbo, Phys. Rev. Lett. 102, 121301 (2009) arXiv:0811.1989 [hep-th]]; N. Kaloper, A. Lawrence and L. Sorbo, JCAP 1103, 023 (2011) arXiv:1101.0026 [hep-th]].

[27] L. Smolin, Phys. Lett. B 93, 95 (1980).

[28] S. Ferrara, D. Lust, A. D. Shapere and S. Theisen, Phys. Lett. B 225, 363 (1989).

[29] I. Ben-Dayan and R. Brustein, JCAP 1009, 007 (2010) arXiv:0907.2384 [astro-ph.CO]]; S. Hotchkiss, A. Mazumdar and S. Nadathur, JCAP 1202, 008 (2012) arXiv:1110.5389 [astro-ph.CO]].

[30] M. Biagetti, M. Fasiello and A. Riotto, Phys. Rev. D 88, 103518 (2013) arXiv:1305.7241 [astro-ph.CO]]. 